\title{
File Transfer in FANET using Dijkstra Algorithm
}

\author{
Alisha Gupta ${ }^{1}$, Sumit Miglani \\ ${ }^{1,2}$ Thapar University, Computer Science and Engineering Department, Patiala, India
}

\begin{abstract}
Many researchers have been attracted on the new research area i.e. flying adhoc network (FANET) after the lot of research done on the mobile adhoc network(MANET). MANET and VANET cannot tackle all the situations so it can be done by the new introduced concept FANET. FANET is the adhoc network which is formed by the multi UAVs instead of using one large UAV. Due to rapid topology change and high mobility of the nodes it is difficult to apply suitable routing protocol for the communication among the UAVs. For the communication among the UAVs routing protocols are used. Few routing protocols can implement the FANET application in real world scenario. The FANET application has not been widely used because there is no protocol for connection establishment of FANET and there are lots of hurdles in deploying FANET. In this paper we discussed the feasibility of deploying FANET in real world environment. A dynamic FANET network topology is created. For the communication among the UAVs the Dijkstra Algorithm is used.
\end{abstract}

Keywords: UAVs, FANETs, Routing Protocol, MANET

\section{Introduction}

Due to the rapid change in technology advancement on electronic, sensor and communication technologies, unmanned Ariel vehicle System have been introduced which fly autonomously or operate remotely without carrying any human personal. Instead of using one large UAV use of multi UAVs is of greater use. In single UAV system communication is done using ground base or satellite is used[1]. In case of communication in single UAV it is done between the UAV and the infrastructure. Using many single UAVs leads to the use of multi UAVs. In multi UAV system the UAVs can be connected to the ground base or the satellite as in single UAV system. The variants of star topology are use for this. UAV to UAV communication can also be done through infrastructure [2]. Various design problems are there for infrastructure based approach. For this each UAV should be equipped with complicated and expensive hardware to communicate with the ground base or the satellite. Due to change in the moment of nodes and the topology changes frequently there is the problem of communication of UAV and the ground station [1]. If the UAV is out of the range with the ground base it disconnects. Multi UAVs have unique challenges basically in case of communication. Capability of single UAV is much less than the multi UAVs. The advantages of multi UAV is summarized as below:

1) Cost: Cost of maintaining the small UAV is less than the large UAV [3].

2) Scalability: With the use of large UAV only limited amount of coverage increases. Multi UAV increases the scalability of operation easily [2].

3) Survivability: If the UAV fails in the mission which is operated by one UAV then the mission stops. If multi UAV is carrying out the operation and one UAV stops working then the operation is carried out by other UAV [2].

4) Speed Up: Mission is completed faster by multi UAV than the one large UAV [3].

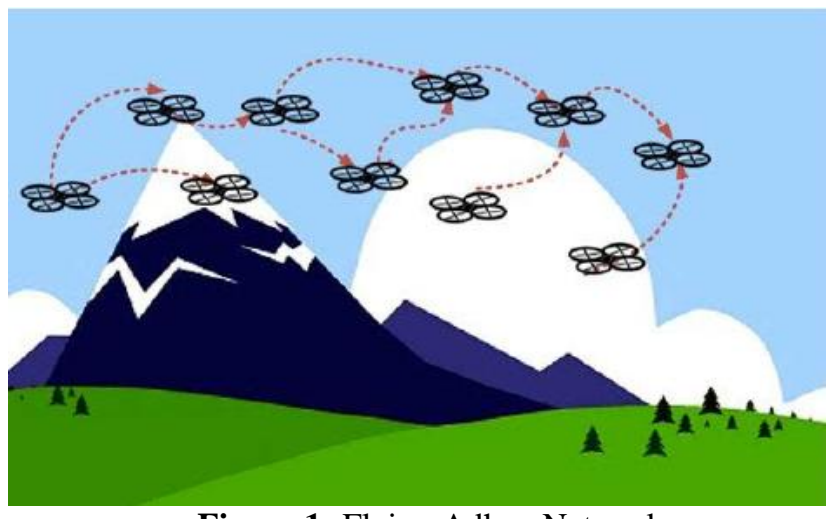

Figure 1: Flying Adhoc Network

An alternative solution is to build an adhoc network between the UAVs which is called Flying adhoc network [4]. UAVs acts as nodes and the nodes are highly mobile due to which the topology changes very frequently. So there is problem of communication among the UAVs [5].For communication among the UAVs many routing protocols have been proposed. The static routing protocols like OLSR, DSDV, AODV which are the communication protocols for MANET and VANET can be used in FANET [6]. There are various problems in implementing Flying Adhoc Network in real world. Due to frequent topology changes and mobility of nodes there is difficulty in implementing FANET with basic routing protocols. For this dynamic routing protocol can be used which can create nodes dynamically according to topology change. In our proposed approach each node can easily communicate with the other node through dynamic network. The remaining paper is structured as follows: Section II gives the brief discussion on the problem and our contribution in resolving it. In Section III we will discuss the work done till now. In Section IV we will discuss the methodology of the research done. Section V discusses the results of the work done. Section VI discusses the conclusion.

\section{Problem Formulation and Contribution}

FANET is the flying adhoc network which deals with the communication between the UAVs. FANET creates a network which contains many UAVs. The mobility of the 


\section{International Journal of Science and Research (IJSR) \\ ISSN (Online): 2319-7064}

Index Copernicus Value (2015): 78.96 | Impact Factor (2015): 6.391

UAVs is much higher than the mobility of the MANET and VANET nodes. The mobility is higher due to which the topology also changes frequently [2]. Due to frequent topology change and high mobility of nodes the application of FANET network in real world scenario is difficult. There are many routing protocols which can be used to simulate the FANET network [6]. But the simulation with different routing protocol does not help with the problem of implementing FANET in real world applications. For better communication there is a need of forming a dynamic routing protocol. Due to this Dijkstra Algorithm is used to find out the shortest path and to make the communication among the network fast and easy.

\section{Related Work}

Ilker Bekmezci et al proposed that communication is the most important design problem in the multi UAV which is the coordination and collaboration between the UAVs. Multi UAV system capabilities are restricted on the infrastructure based architecture. If the UAVs are connected to the satellite or ground base then the communication is only on the basis of infrastructure. Adhoc networking is the way to solve the problem arising with the infrastructure based approach. Flying adhoc network (FANET) is the adhoc network which connects the UAVs [2].

Naveen et al proposed that the UAVs capabilities have evolved rapidly and is used in military and civilian areas. One of the main issue in multi UAVs is the communication between them. Flying adhoc network solve this problem easily. The main design issues and challenges in the flying adhoc network is introduced [1].

Md. Hasan Tareque et al proposed the comparative study of FANET with the tradition networking models like MANET and VANET. The traditional routing protocols are categorized into six methods which are analyzed and compared on the basis of performance criteria. The comparative analysis helps in choosing the best routing algorithm where FANET can be deployed [3].

Kuldeep Singh et al proposed that due to high mobility of the FANET nodes and the frequent topology change the routing algorithms are used. Adhoc networks performance is greatly enhanced by routing protocols.In this paper the experimental analysis is done on AODV, DSDV, and OLSR routing protocol using ns 2 . The comparative study is done on the basis of three parameters packet delivery ratio, end to end delay and throughput [6].

Kuldeep Singh et al proposed that the performance of routing protocol is optimized by mobility models. The research aims in implementing the OLSR routing protocol in FANET and studying the OLSR in different mobility models and to optimize the OLSR in FANET on the basis of three parameters packet delivery ratio, end to end delay and throughput in ns2[8].

Ganbayar Gankhuyag et al proposed that due to dynamic environmental conditions, terrain structures, high mobility the routing protocols of mobile adhoc network are not suitable for flying adhoc network. Directional and omnidirectional transmissions are combined and used in FANET using a novel directional routing scheme. Location and trajectory information is used for geocasting and unicasting routing with proposed scheme. Route setup success rate and average path lifetime is increased with this method than the traditional AODV method [9].

Farhan Mohammed et al proposed that to protect the related missions and services to be attacked in intentional and unintentional manner the trusted strategies and protocols should be used. MANET is the collection of autonomous nodes with the dynamic topology. There are common characteristics between the UAVs and MANET. Individual UAV is node but the group of flying UAVs is MANET. The trust based protocols and management schemes are discussed which can be used in UAV networks and the UAV applications where this can be used [10].

Denis Rosario et al proposed as the topology changes rapidly so reliable and robust routing service for FANET must be used. In case of buffer overflow and the packet loss also the user experience on watch live video streaming should be satisfactory. Geographical-aware beaconless opportunistic routing protocol (XLinGO) and Cross Layer Link quality are introduced in this paper. This helps in creating and keeping reliable multi hop routes and by this enhancing the transmission of multiple video flows over FANET. For routing decisions XLinGO considers the human based information as performance metrics and Quality of Experience. Quality of Experience support multi hop, multi flow and multimedia dissemination is achieved by XLinGO using performance metric [11].

WajiyaZafar et al proposed that due to complex protocols for communication in FANET the cost of COT components, high mobility and computational resources, limited radio bandwidth maintaining the QoS has become difficult task. To reduce the communication cost, optimize the network performance and optimize network performance the new scheme is proposed of multicluster FANETs for efficient network management .Using GTS and TDMA both the beacon enabled and beaconless modes are investigated. The bandwidth is reserved for latency critical applications, eliminate collisions and medium access delay are done with this proposed methodology. The routing protocols like OLSR, DSDV, and AODV are also analyzed. High packet delivery ratio is guaranteed with this scheme and the acceptable level of latency requirements are maintained compared with the complex and dedicatedly designed protocols [12].

In 2015 Stefano Rosati et al reported the routing protocols are compared for adhoc network i.e. optimized link state routing (OLSR) and predictive OLSR (P-OLSR). P OLSR is the designed extension of OLSR for the FANET which uses GPS information. The Linux implementation is available only for P-OLSR. The results of both real world experiment and the Media Access Control (MAC) layer are presented. A test bed is created of two fixed wing UAVs and a node on the ground. The communication range, routing performance and the performance are evaluated through this experiment. P-OLSR is better than the OLSR in frequent topology changes [7]. 


\section{International Journal of Science and Research (IJSR) \\ ISSN (Online): 2319-7064}

Index Copernicus Value (2015): 78.96 | Impact Factor (2015): 6.391

\section{Methodology}

In our Methodology a FANET network is created comprising of a Single Server node and few client nodes as shown in figure 2. A Single Server node acts as the base station to the network that will keep the track of all the client nodes that act as the UAVs in the network and in addition to it will send the IP address of each client to its neighboring client nodes that act as routers for the communication purpose. Out of it, one of the UAV is selected as the source node and other UAV can be selected as the destination node. The UAV which acts as the sender will select the message that it wants to send to the receiver. The message selected could be anything audio, video, text, image etc and after it the Dijkstra algorithm will run to find the shortest possible path to the destination node.

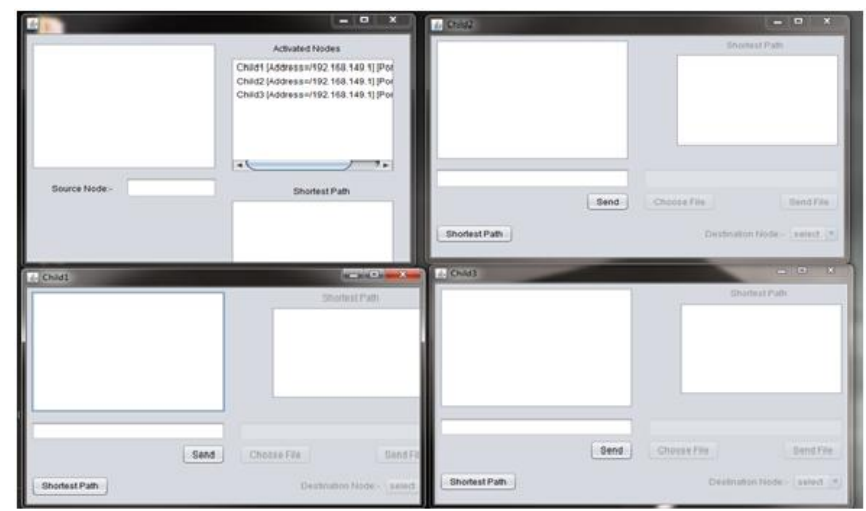

Figure 2: Template showing the experimental method with base station and UAVs

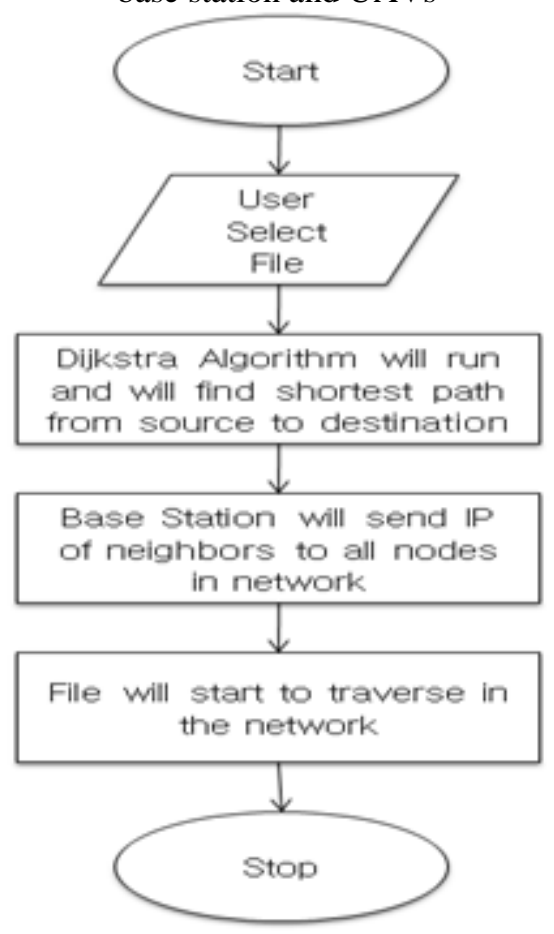

Figure 3: Flow Chart

\section{Experimental Results}

For communication in the FANET network between the source node and the destination node the file is being transferred. From all the nodes the source node and the destination node is selected. Three client nodes (UAVs) are shown in Fig 4 along with a server node (base station). The activated client nodes are shown in the server node. In Fig 5 the reply is received from the activated nodes. The Source node is selected from the client nodes and the other activated nodes will be shown in the server node from which the destination node can be selected. Fig 6 is showing that the destination node is selected at the source node. The file transfer will take place among those two nodes by taking the shortest path. Fig 7 shows that the on the source node the file can be selected which we want to transfer. The dialog box appears showing all the files. Fig 8 is depicting that the file is transferred from the source node and it takes the shortest path using the Dijkstra algorithm. The file will start to traverse and will reach the node which falls in the shortest path. In Fig 9 the file finally reaches to the destination node. Hence the file transfer is successfully done in the FANET network using Dijkstra Algorithm.

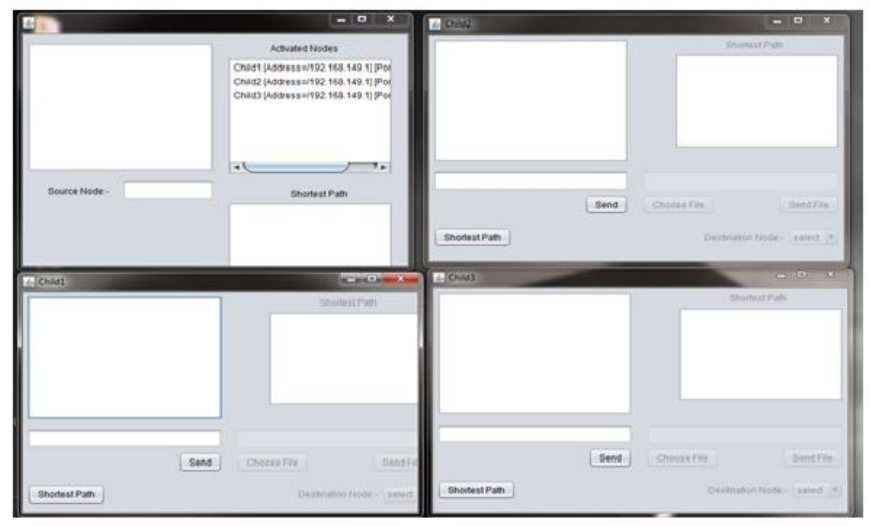

Figure 4: Activated Nodes

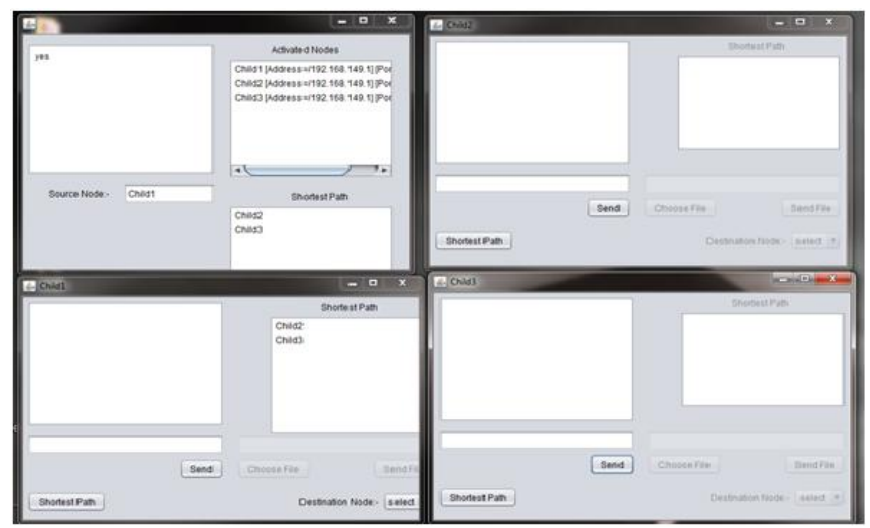

Figure 5: Reply from the child nodes

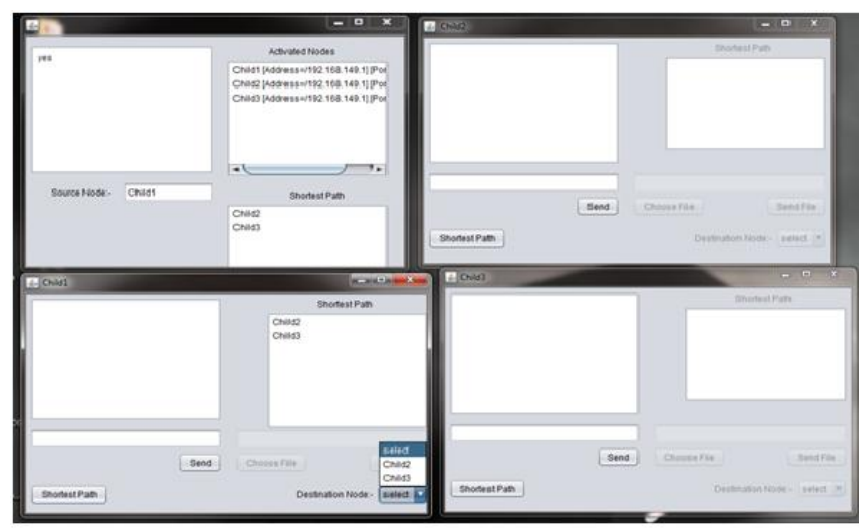

Figure 6: Destination node selected 


\section{International Journal of Science and Research (IJSR) ISSN (Online): 2319-7064}

Index Copernicus Value (2015): 78.96 | Impact Factor (2015): 6.391

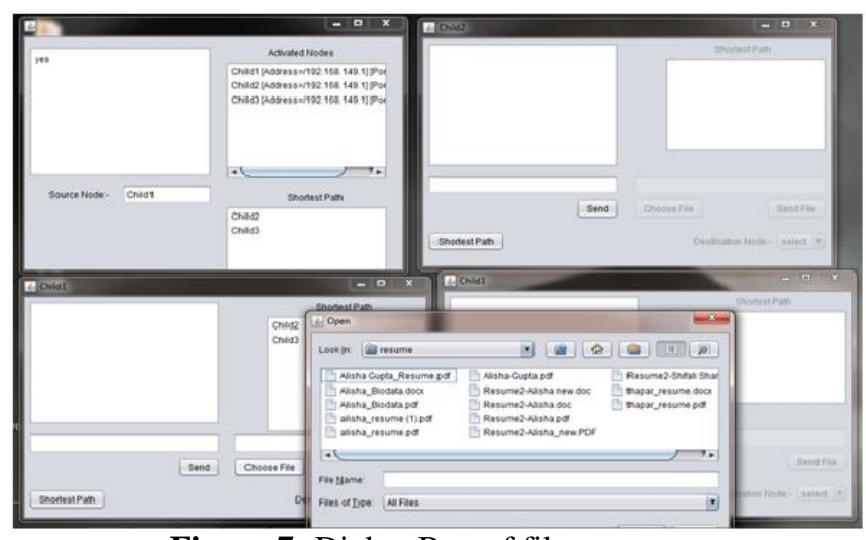

Figure 7: Dialog Box of files appears

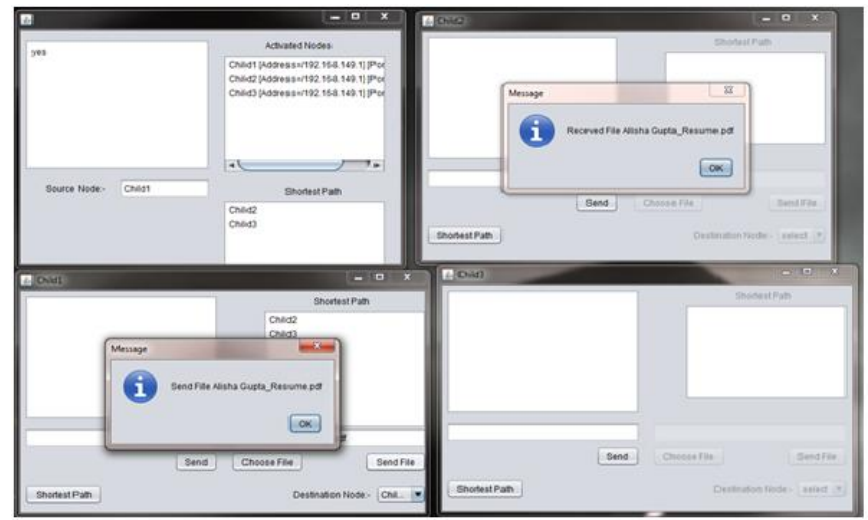

Figure 8: File received by node on the shortest path

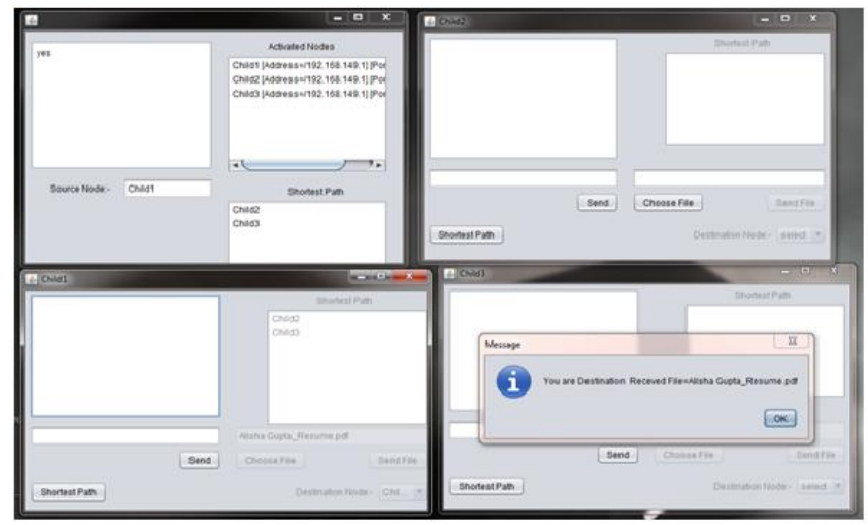

Figure 9: File received by Destination node

\section{Conclusion}

In this paper the FANET network is made dynamically to show its implementation in real world using the server node which is the base station and 3 client nodes which acts as UAVs. As the mobility of the UAV nodes is very high so the topology changes frequently so FANET implementation in real world application is difficult. The communication between the UAVs is considered as the main problem in FANET. For fast communication this method is proposed. The Source node has to send the data to the destination node so for that shortest path is required. The shortest path is calculated using Dijkstra Algorithm. The Base station will send the IPs of neighbors to all the nodes in the network. Thus for sending any file from source node to destination node that shortest path is followed. The file will start to traverse moving to all the nodes in the shortest path.

\section{References}

[1] Sunil Maakar and Naveen, "Concept of flying ad-hoc network: A survey", pages 2349-7688, 2015.

[2] Ilker Bekmezci, Ozgur Koray Sahingoz, and amil Temel, "Flying ad-hoc networks (fanets): A survey", Ad Hoc Networks, 11(3):1254-1270, 2013.

[3] Mohammed Atiquzzaman, Md. Hasan Tareque, Md. Shohrab Hossain, "On the routing in flying ad hoc networks", 5:1-9, 2015.

[4] Simarjot Kaur and Meenu Talwar, "Routing strategies in flying ad-hoc networks", Journal of Network Communications and Emerging Technologies(JNCET) www. jncet. org, 6(3), 2016.

[5] Karan Palan and Priyanka Sharma, "Fanet communication protocols: A survey", Volume 7:219223, 2015.

[6] Kuldeep Singh and Anil Kumar Verma, "Experimental analysis of aodv, dsdv and olsr routing protocol for flying adhoc networks (fanets)",In Electrical, Computer and Communication Technologies (ICECCT) 2015 IEEE International Conference, pages 1-4, IEEE, 2015.

[7] Stefano Rosati, Karol Kru zelecki, Gregoire Heitz, Dario Floreano and Bixio Rimoldi, "Dynamic routing for flying ad hoc networks", IEEE Transactions on Vehicular Technology, 65(3):1690-1700, 2016.

[8] Kuldeep Singh and Anil Kumar Verma, "Applying olsr routing in fanet", In Advanced Communication Control and Computing Technologies (ICACCCT), 2014 International Conference, pages 1212-1215. IEEE, 2014.

[9] Ganbayar Gankhuyag, Anish Prasad Shrestha, and SangJo Yoo, "A novel directional routing scheme for flying ad-hoc networks", In Information and Communication Technology Convergence (ICTC), 2016 International Conference, pages 593-597, IEEE, 2016.

[10] Farhan Mohammed, Imad Jawhar, Nader Mohamed, and Ahmed Idries, "Towards trusted and efficient uav-based communication", In Big Data Security on Cloud (BigDataSecurity),IEEE International Conference on High Performance and Smart Computing (HPSC), and IEEE International Conference on Intelligent Data and Security (IDS), 2016 IEEE $2^{\text {nd }}$ International Conference, pages 388-393. IEEE, 2016.

[11]Denis Rosario, Zhongliang Zhao, Torsten Braun, Eduardo Cerqueira, Aldri Santos, and Islam Alyafawi, "Opportunistic routing for multiflow video dissemination over flying adhoc networks", In A World of Wireless, Mobile and Multimedia Networks (WoWMoM), 2014 IEEE $15^{\text {th }}$ International Symposium, pages 1-6. IEEE, 2014.

[12] Wajiya Zafar and Bilal Muhammad Khan,"A reliable, delay bounded and less complex communication protocol for multicluster fanets", Digital Communications and Networks, 3(1):30-38, 2017.

\section{Author Profile}

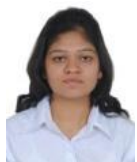

Alisha Gupta holds the Bachelor of Technology (B.Tech) degree in Information Technology from PTU, Jalandhar, India (2015). She is pursuing Master of Engineering degree in Information Security from Thapar University, Patiala, India (2017). Her research 


\section{International Journal of Science and Research (IJSR) \\ ISSN (Online): 2319-7064}

Index Copernicus Value (2015): 78.96 | Impact Factor (2015): 6.391

interests include Network Security, Ethical Hacking, Software Engineering, Wireless Network, and Adhoc Networks.

Sumit Miglani is Assistant Professor in Computer Science and Engineering Department, Thapar University, Patiala, India. He holds a Bachelor of Technology (B.Tech.) degree in Computer Science and Engineering from PTU, Jalandhar, India (2002). He obtained his Master of Technology degree in Information Technology with specialization in Wireless Communication and Computing from Indian Institute of Information Technology (IIIT), Allahabad, India (2007). His research interests include Wireless network, Mobile Adhoc Network, Flying Adhoc Network, Vehicular Adhoc Network, Software Engineering, Programming Languages and Mobile Agents Applications. 